\title{
FLUFFY AODV BASED DYNAMIC LOAD BALANCING ROUTING TO IMPROVE PERFORMANCE AND DATA AVAILABILITY IN MANETS
}

\author{
M. Mahto \\ Govt. Polytechnic, Korba,Chhattisgarh, India \\ msmahto@yahoo.co.in \\ Neelam Sahu \\ Associate Professor, Department of IT and CS, \\ Dr. C.V. Raman University, Kota, Bilaspur, Chhattisgarh, India \\ neelamsahu16@gmail.com
}

\begin{abstract}
Mobile Ad Hoc Networks (MANET) supports portable communication among host devices in flux. These networks have no set architecture, that ensures that the routing of packets can be optimal under multiple factors, for example by increasing the communication within nodes, which dramatically increasing the number of nodes within an area. In the field of fast-moving nodes that result in their decreased lifespan, MANETs often have low stability. Ad hoc on-demand distance vector (AODV) routing protocol is an ad hoc, descriptive on-demand routing protocol. In this paper, standard AODV protocol is modified to important node measurements called Fluffy Ad Hoc On-Demand Distance Vector (AEAODV).This proposed techniqueutilizes fluffy rationale procedures to decide a nodes trust an incentive by joining the remaining energy also, speed of every node in the MANET to achieve this improvement. Simulation research shows that AEAODV improves network throughput \& improved network overall efficiency in terms of increased packet delivery, decreases overhead \& jitter control, increases overall throughput, decreases interferences as well as, finally, improves the end-to-end delay in medium to high-density terms.

Keywords: MANETs; Aggregation; Traffic Congestion;AODV;Fluffy AODV.

\section{Introduction}

MANETs nodes are autonomous [Garg et al. (2017)] [Murthy et al. (2009)]. The scenario of MANET mobility management is shown in fig. 1 [www.researchgate.net,(2021])]. The shortest route often does not ensure a safe route because of the highly complicated behavior of nodes. The reliability of the route is also not factored because of the route in a complex MANET can easily be broken[Bento and Wille (2020)].It is therefore of high significance to find a stable and trustworthy route. MANETs focus on the collaborative as well as trusting nature of nodes for their integration as well as survival. In the contrast, the existing routing protocols (RPs) generally presume that every node in a network is dependable as well as cooperative. In the disaster \& relief, mobile surveillance \& military operations, MANETs will be used.
\end{abstract}

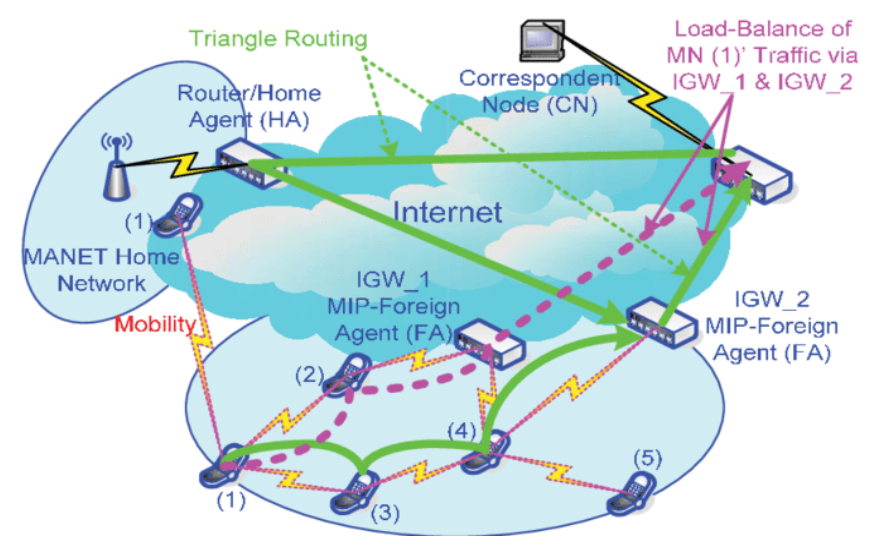

Fig.1. A scenario of MANET mobility management 
MANET routing is very complex because of node movement [Akhtar et al. (2017)]. Predicting the location and routing of nodes based on expected locations that help to create a long-term routing path. The number of predictions is focused on the previous node's locations. The significant messages that may create congestion to block transmission of emergency messages are exchanged during the exchange of information. The avoidance of congestion [Garg et al. (2018)] is a big challenge thatgiven mobility, and dynamism of MANET topology. Fig. 2 [https://ars.els-cdn.com/(2021)] indicates the congestion scenario.

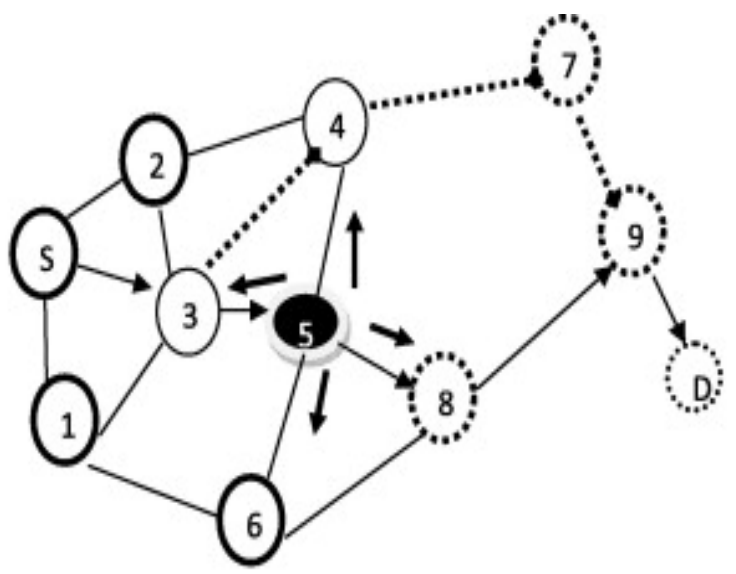

Fig. 2. Congestion in MANET

An on-demand method for the configuration of ad RPs is used to overcome the drawbacks of the table-driven method. AODV [Parkinset al. (2003)] is a descriptive on-demand ad-hoc RPs. In AODV, the creation of routes only occurs when new routes are demanded. Unicast, broadcast \& multicast routing are available from AODV. AODV will quickly respond to network topology changes \&modified only hosts with RREQ messages to be impacted by network change. The route discovery [Chavanet al. (2016)] is the responsibility for RREQ \& RREP communications. A source communicates an RREQ packet for a path to the destination during the AODV road discovery process. If there is a valid route to the destination of source nodes in its routing process, a transitional node may respond with an RREP packet to RREQ \& disable the RREQ packet. We name this method mechanism for the cached route. A fast configuration time, high probability of route setup as well as limited RREQ packet sizes have been the merits of the route cache mechanism. Even so, there are a certain number of mobile nodes with the demerit of a route cache mechanism [Kim et al. (2005)].

In this paper, we recommend an AODV from a selective routing mechanism depended on a source/destination pair. Following this INTRODUCTION, the rest of the work is prepared as occurs: Section II provides an overview of routing in congested ad hoc, AODV, Section III discusses various research work that has already done by different researchers, Section IV describes the AEAODV proposed method with a model, Section V defines the simulation model, efficiency as well as observational measurements. After this, Section VI includes the conclusion.

\section{Congested Routing in AD Hoc Network}

The routing process (RR) is a path selection process for sending data packets from source to endpoint. RP defines the interaction between routers by selecting the route from one of its 2 nodes. Every router has network data thatis directly connected. The algorithm for routing defines router choice. Routing protocol extracts data from each router as well as spread it throughout the network [Alex et al. (2013)][Santosh and Sachin (2015)].

Packet routing is a key difficulty facing MANETs as a result of unpredictable links, dynamics, as well as limited resources [Boukercheet al. (2011)]. Many MANETs attributes help to complicated the development of ad hoc protocols: density, mobility, heterogeneity of devices, QoS, battery constraints as well as bandwidths. The major issues in mobile communication are the transmission of a static transmission power packet that can drain the interaction interface's energy consumption. Packages thatare transferred at the highest common power create a significant energy loss. Subsequently, the power transmission necessary for the communication of node pairs may be maintained at the minimum, if they are close to one another.In this scenario, high-power packets can create significant network intervention as well as consume more than necessary power. There are many difficulties to routing such as delays, jitters as well as overhead interaction due to node mobility. In this scenario, whenanode leaves or joins a network, the topology is altered. Routing mechanisms for anappropriatebroadcast of data packets with better rates of delivery are also dependent upon system performance [Krupa and Benakappa (2010)][Anuradha and M. (2017)]. 
Congestion is corrupted QOS where network node carries more data than capability. Mobile nodes are multihop wireless links in ad hoc networks mobile nodes. Since no fix required infrastructure when connects various with each other, that is, when nodes communicate data to the destination, the network's intermediate nodes can overload, because they are larger than linking or network capabilities than number of packets that are sent on wireless links. This overloaded network may lead to a large loss of packets, increased delays, as well as a reduction in network performance on wireless connections [Leemaroselin(2015)][Mamtaet al. (2017)].The congestion may occur because of the dynamic topology of the network wherein E2E connectivity can sometimes be lost because of continuous node movement.

AEAODV routing protocol arises from the DSDV[Parkins and Bhagwat (1994)] \&has been specifically developed for ad hoc mobile networks. AODV explores routes throughout the network through the flooding of a message. There arefour kinds of messages like RREQ, RREP, RERR\& Hello available in AODV as a whole. PREQ is a route discovery message, RREP message recovers when another route or any path intermediary node is found. The RERR message is being used in the instance of connection failure by the node presenting link failure. Send RERR communication back to the source node and initiate a path discovery from the source node again. AODV uses a loop control destination address. AODV uses number of flags \& fields of RREQ message in its route discovery phase. It included source $\&$ destination node names, source/destination sequence numbers, significant cost identification numbers as well as other validation flags. The source ID for RREQ defines route request which is utilized to avoid the similar message from being replicated. Once the entire data on that route is detected, in simple AODV, the entire information is transmitted before the data is not connected or transmitted. This tool is appropriate when transmission involves a connection for a shorter period when the transmission is required. The standard flexibility characteristics in MANET may be efficient for small transmissions requiring a long-term connection. New effective routes are identified from time to time during transmission instead of sending whole data along a single route. [Ahmad and ur Rahman (2010)].

\section{Literature Survey}

The key aim of a network RP is to efficiently manage packets as well as deliver them to their final destinations. Mobile ad-hoc network RPs are distinct from those for wired networks which expecting to find at decreasing the number of transmissions to shorten the delay in the end to end, instead of some other parameter including performance. RPs are also categorized as proactive or reactive in these network types. Proactive protocols try to keep routes to all network nodes, while reactive protocols create only routes as required for transferring data at this point. [Shukla et al. (2013)] suggested a new AODV routing simulation methodology. The methodology suggested is based on the random walk model of Markov. They quantify the distribution of probability for some communication operations. This study helps to explain how the different component relies on the AODV and how this impactsQoS. Its performance and effectiveness were measured with different criteria as well as reliability including scalability were further analyzed. [Singhet al. (2014)]discussed routing protocols tested for the ad-hoc network have varied CBR traffic but have common mobility as well as stationary conditions. GloMoSim simulator findings after comparing various ad-hoc routing protocols AODV, DSR, BFD as well as WRP, regardless of performance, collision as well as byte loss, The outcomes of the simulation as well as the analysis are described in detail. For any network, an acceptable, as well as best choice of protocol, is a little complicated. Like illustrated in the graphs, AODV, DSR routing protocol operates more reliably, combining performance indicators with performance metrics with collision. [Pal and Dutta (2015)] used AutoreGressive Integrated Moving Average (ARIMA) as well as MLP models to determine the path length between the origindestination pair in MANET. MANETs extract the path-length data utilizing three different models: the Manhattan Grid Mobility Model (MHG), ii) Random Way Point (RWP) as well as (iii) Reference Point Group Mobility Model (RPGM). The predictive precision of the path length determination of AODV routing between source and destination nodes was evaluated by this analysis. NNs could be used better than mathematical models in the projection of path length between mobile nodes \& NN models.[Ferdous and Hossain (2016)] recently proposed ideas for MANET wireless ad-hoc network congestion control problem. To quantify the drop factor, changes to the RREQ package were made. The current technology device was simulated with the 2.35 network simulator. The consequence is that, about package availability, throughput as well as latency, the suggested solutions have done relatively better than standard AODVs. [Sarkaret al. (2018)] proposed route selection mechanism together with the AODV Protocol to boost QoS MANET is introduced. The finest route is chosen for data transmission using the pheromone value of path-dependent on ant colony system by AODV. pheromone value of route is dependent upon the path reliability, congestion, hop number as well as the majority of the nodes along the path in the future framework. For data packet transference the route with the highest pheromone value is chosen. The findings reveal that the suggested scheme exceeded AODV routing algorithms, DSR \& Enhanced-Ant-DSR.[Bamhdi(2020)] offered a method of dynamically changing the AODV standard protocol to the power used for transmission, called Dynamic Power-AODV (DP-AODV). To accomplish this increase, this study provides the reliance on the density of a transmission spectrum. The study found that DPAODV demonstrates a decrease in delay than AODV with increased density overall enhanced efficiency for 
densely populated networks over 200 nodes. The findings of the simulation demonstrate that DP-AODV improves network efficiency while decreasing node interruption in the dense region, \& increasing network inclusiveefficiency relative to improved packet delivery, reducing overhead as well as jitter controls, increasing overall output, reducing interference as well as, ultimately, scaling costs medium- to high-density end-to-end retardation. [Yadavand Firdaus (2019)] discussed limited bandwidth and changing topology results in the problem of Congestion in MANETs. Detecting Congestion is difficult in a wireless network, since there may be several reasons behind the dropping of packets. In a movable network, failed delivery could be due to the route break as well because of the mobility of nodes. So the cause behind packet loss is not only Congestion. Congestion-aware routing is done by using the clustering approach. The Mobile Clustering (MOBIC) approach of MANET is used for clustering. This is based on the mobility characteristic of the node present in MANET. It improves packet delivery ratio \& also shows improvement in E2E delay when associated with normal (AODV) Routing of MANET. [Aouizet al. (2019)] suggested a different network centrality parameter calculation focused on the channel busyness ratio. The current CBMLB protocol strengthens the current AOMDV multi-path protocol. By spreading load through alternative paths available, the CBMLB tries to minimize central congested nodes that have an elevated channel busyness ratio. The paper protocol depends on disjoint routes to packets that have been less congested. A contrast with efficiency in terms of PDR, delay E2E, including overhead connectivity, with AODV, AOMDV \& QMLB protocols. [Saba Farheenand Jain(2020)] discussed and determine the possible locations of the nodes using a hybrid model, a forecast was based mainly on spatialtemporal findings related to its region. To increase the routing efficiency without a bigger packet overhead is suggested multi-path RP centered on predicted possible places with path diversion in required places along the path.[Balakiruthigaet al.(2020)] suggested software-defined network infrastructure segment-dependent electricity routing method aims to optimize the performance by ensuring residual capacity as well as proper connection usage. The method also indicates a reduction of the label stack length concerning the highest depth of the segment label. The study is carried out by comparing other modern practices to their energy information routing method in the distributed atmosphere in one single supervisoratmosphere. A single failure point prevents the distributed controller setup from networking. It helps deter overhead operators as well as improves application performance through utilization.

\section{Proposed Methodology}

The proposed methodology is divided into three section like problem statement, proposed method and proposed algorithm.

\subsection{Problem Statement}

In conventional AODV, base number of bounces metric is utilized to settle on a choice about course choice, be that as it may, this is certainly not an adequate boundary for building the best course to an objective in a remote MANET. It doesn't consider different components that may impact course quality, for example, got signal strength, node portability, or node leftover energy, amongst others. We have introduced in this paper a fluffy AEAODV protocol to improve the existing AODV routing Protocol.

\subsection{Proposed Method}

In the proposed fluffy AODV (AEAODV), significant node measurements, for example, node remaining energy and versatility are considered to develop a solid course and limit the likelihood of course disappointment during information parcel transmission. The decision of reliable nodes used to construct a steady course in our fluffy calculation depends on nodes that have higher lingering energy levels\&transfer with the least speed.

The proposedmethod utilizes fluffy rationale procedures to decide node's trust an incentive in joining remaining energy also, speed of every node in MANET. The nodes with the most noteworthy trust esteems are chosen to set up the best course accessible to an objective node. Each middle of road node figures its trust esteem at whatever point it gets the RREQ parcel. The halfway node starts a clock if the RREQ parcel has not been recently gotten. During the clock length, the middle of the road node gets more RREQ packets (of a similar recognizable proof ID and succession no.) from its neighbors. moderate node chooses node with best trust esteem (conveyed by got RREQ packets) to refresh its converse course table, that will be utilized to build opposite unicast course as a piece of a solid course foundation among source what's more, objective. After the clock terminates, the middle of the road node advances the RREQ, conveying the transitional node's trust as an incentive to different neighbors. The clock is utilized to inspect very RREQ packets that show up at various occasions to the middle of the road node, and at that point, one with the most elevated trust esteem is sent. This method to choose the best way to utilize reliable nodes limits the measure of overhead control packets 
overflowed all through traffic \& decreases the likelihood of traffic congestion. Fig.3 is explaining the whole process.

\section{Proposed Algorithm}

IF-THEN rule analysis, result aggregation as well as evaluation of the value of logic algorithm description. The following steps involved in this research:

Step 1. Start

Step 2. Take input parameter values and convert into probabilistic values. In our case, the input parameters are node residual energy, node speed as well as hop count number as well as threshold calculation.

Step 3. Evaluation of IF-THEN conditional statement.

Step 4. In Step 1 the threshold levels are fed to IF-THEN for the condition of the output range. AND operator selects from the values defined the minimum threshold values.

Step 5. In this stage, output aggregations, the method gathers all outputs in union form that outcome from IF-THEN \& then uses OR operator to select max evaluation value to construct a new aggregate threshold set.

Step 6. Convert probabilistic values into actual values and the centroid method is implemented to measure the node trust value for the current aggregate function reached at step 4.

Step 7. Calculate all performance metrics

Step 8. Obtain improved performance of a network

Step 9. End

RREQ (x) from node

(k) with Hop count=1

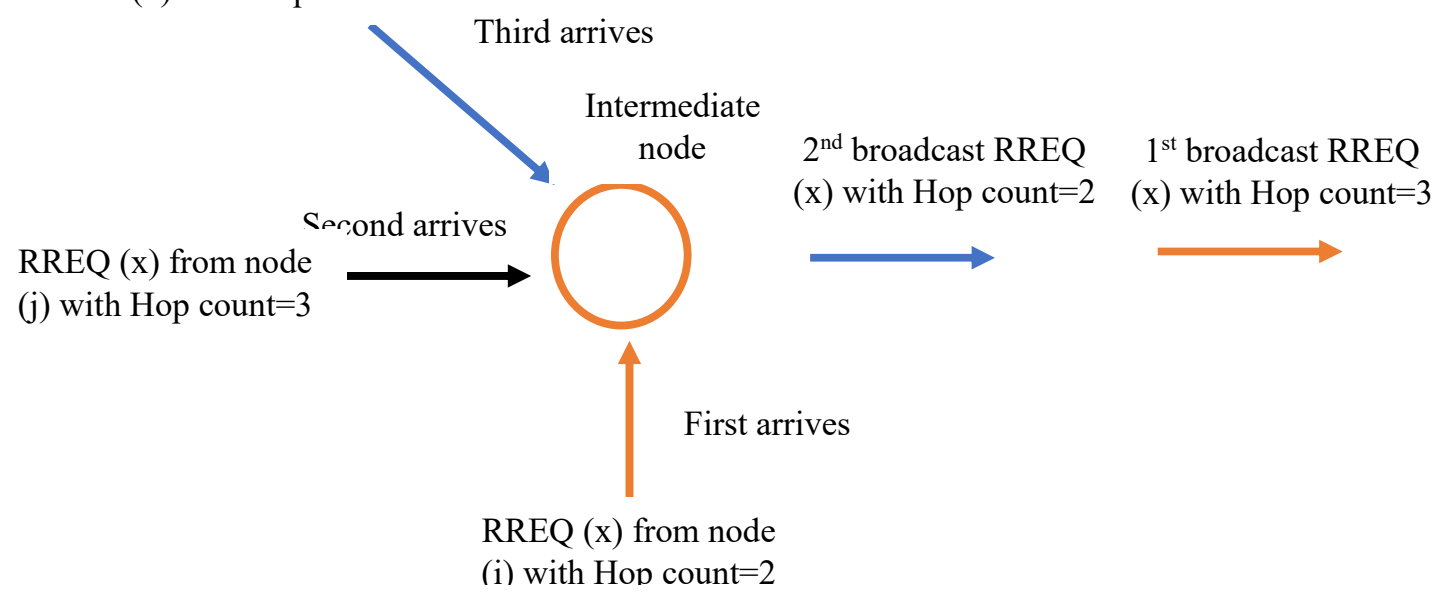

Fig.3. Intermediate node RREQ broadcasting in classic AODV 


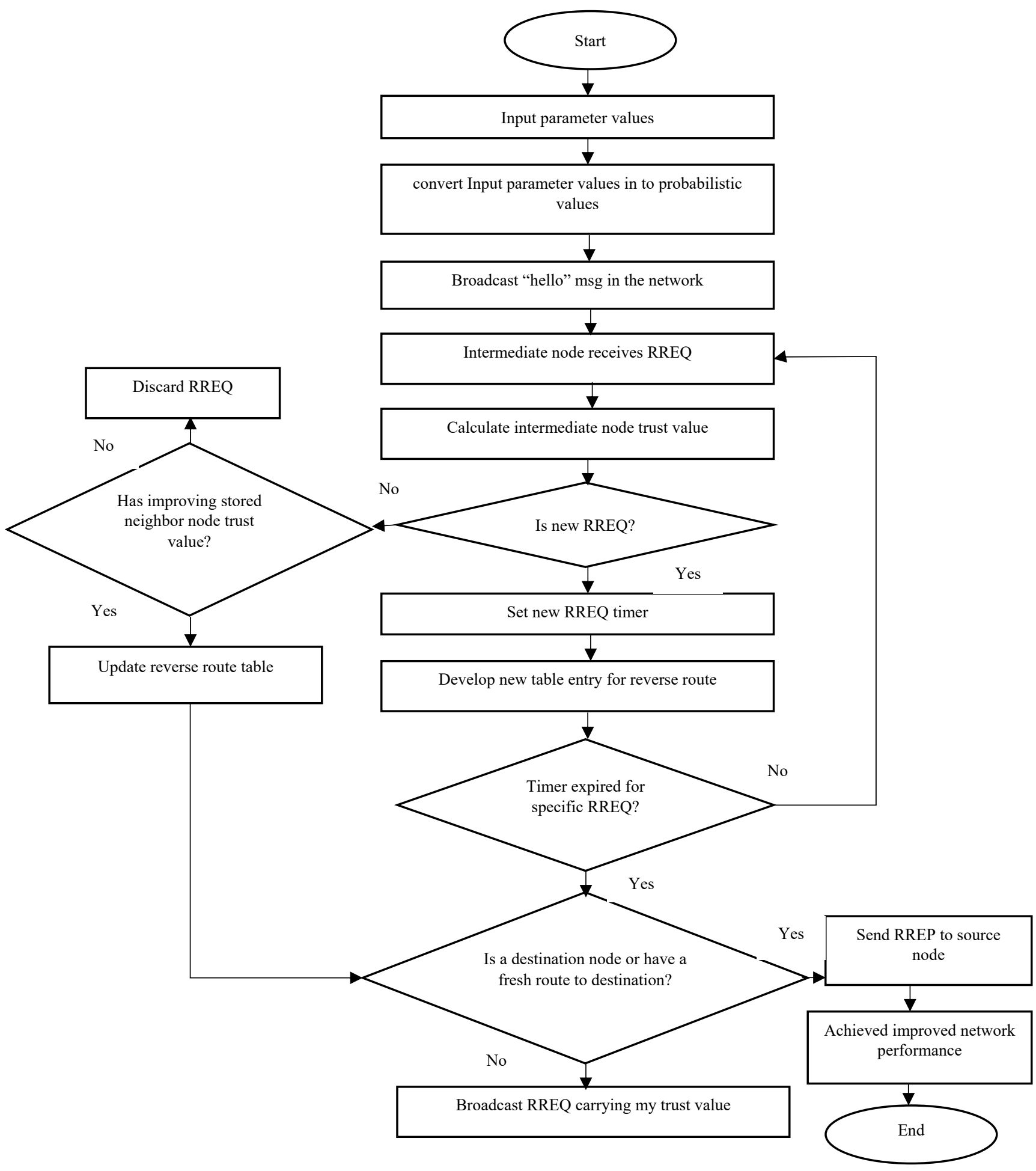

Fig.4. Flowchart of fluffy-based trust value computations (AEAODV)

\section{Simulation Results and Analysis}

In our research, we have utilized a Network Simulator 3 (NS-3) and that's extremely common in the technology, research \& design community in data communication and computer networking. It is an event-oriented method for simulating the AEAODV communication protocol to be studied and evaluated. The protocols such as AODV, DSDV, OLSR, and EAODV are supported. It is performed on different simulation parameters given in Table I. 


\begin{tabular}{|c|c|}
\hline Parameter & Value \\
\hline Maximums number of host & $50-70$ \\
\hline Movement Patterns & Constant Bit Rate \\
\hline System Size $\left(\mathrm{X}^{*} \mathrm{Y}\right)$ & $1000 \times 1000$ \\
\hline Reproduction Time & $0-100$ and $100-200 \mathrm{~s}$ \\
\hline communication Packet Rate & $20-30 \mathrm{~m} / \mathrm{s}$ \\
\hline Interruption Time & $1.0 \mathrm{~s}$ \\
\hline Directing Protocol & AODV/EAODV/AEAODV/O $/ \mathrm{DSDV}$ \\
\hline MAC Protocol & $802.11 \mathrm{~b}$ \\
\hline Channel Data Rate & $11 \mathrm{Mbps}$ \\
\hline Versatility Speed & $10 \mathrm{~m} / \mathrm{s}$ to $50 \mathrm{~m} / \mathrm{s}$ \\
\hline
\end{tabular}

Table 1. Simulation Parameters

\subsection{Performance Metrics}

Many metrics could be used to increase the performance, but the following metrics were presented for the evaluation of routing protocols for this study:

- Packet Delivery Ratio (PDR):ThePDR through CBR is decreased by continuous bit rate source on destination over sent packets (CBR, application layer). The metric shows us the efficiency of protocol.

- End-to-End Delay (E2E):Cumulative delay caused by buffering when routes that stand at interfaces are identified, retransmission delayed at the MAC as well as the time needed for propagation as well as transmission.

- Throughput:The number of the data sent to the destination per unit time (bytes sent per second).

- Control Overhead:The ratio of total control packets to total packet number transfer to MAC layer.

- Jitter:Measure variations in packet time obtained by the endpoint, generally associated with network congestion, time delays for transmission, time drift, or route modifications for the destination. This occurs mainly in a non-deterministic system.

- Flooding:It is the transmission of a packet router from any node to any other node connected to the router excluding the node from which the packet is received. Flooding involves rapidly distributing modified routing data to each node in a wide network.

\section{Performance Results}

The outcomes of the research demonstrate the benefits of modifications in AEAODV as compared to basic RPs OLSR, DSDV, AODV as well as EAODV. In experimental conditions, the comparison of the results obtained is more useful as well as effective. Different traffic patterns including traffic pattern scenario files have been run on all RPs to allow a fair comparison without bias. There are numerous performance parameters on that simulation results have achieved.

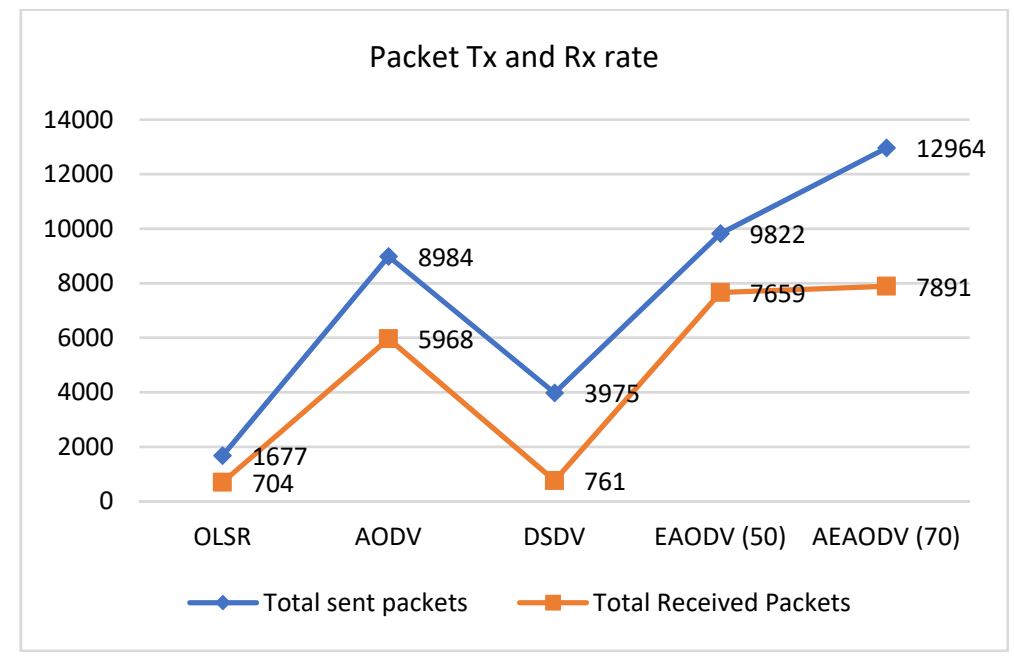

Fig.5. Control overhead by AEAODV with otherRPs

Fig.5 has shown a comparison of different RPs with the proposed AEAODV protocol in terms of the total sent and received packets to measure overhead. From this comparison graph, we can see that the proposed AEAODV 
protocol has higher communication and data transmission rate than other routing protocols. AEAODV sent 12964 packets and received 7891total packets that are higher in comparisonto OLSR, AODV, DSDV, and EAODV.

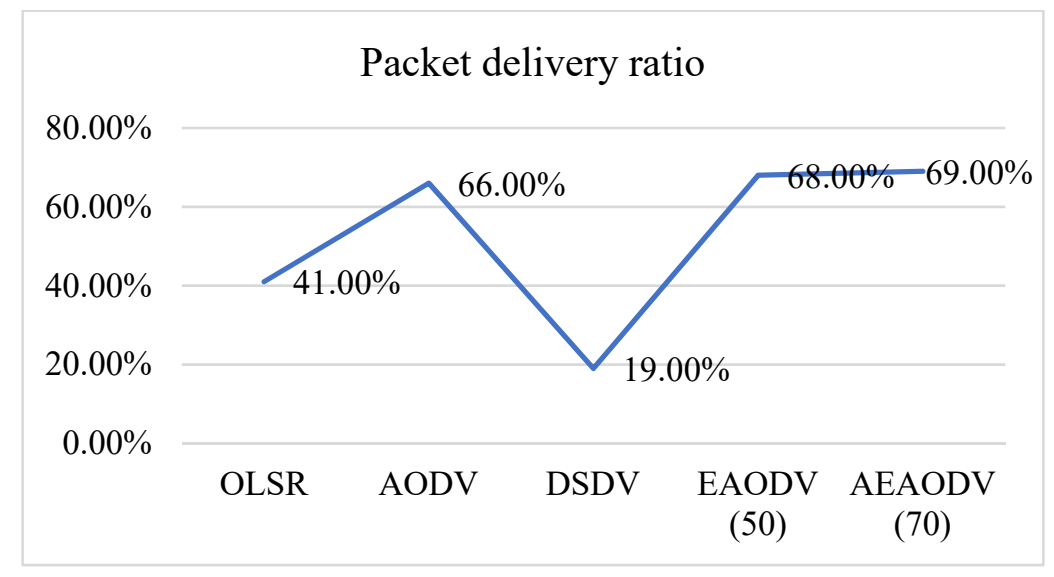

Fig. 6. PDR by AEAODV with other RPs

Fig. 6 has shown a comparison of diverse RPs with the proposed AEAODV protocol in terms of PDR (in \%). The PDR of OLSR is $41 \%$, AODV is $66 \%$, DSDV is $19 \%$, EAODV is $68 \%$, and AEAODV is $69 \%$. From this analysis, it is observed that the proposed AEAODV protocol has higher PDR than other routing protocols.

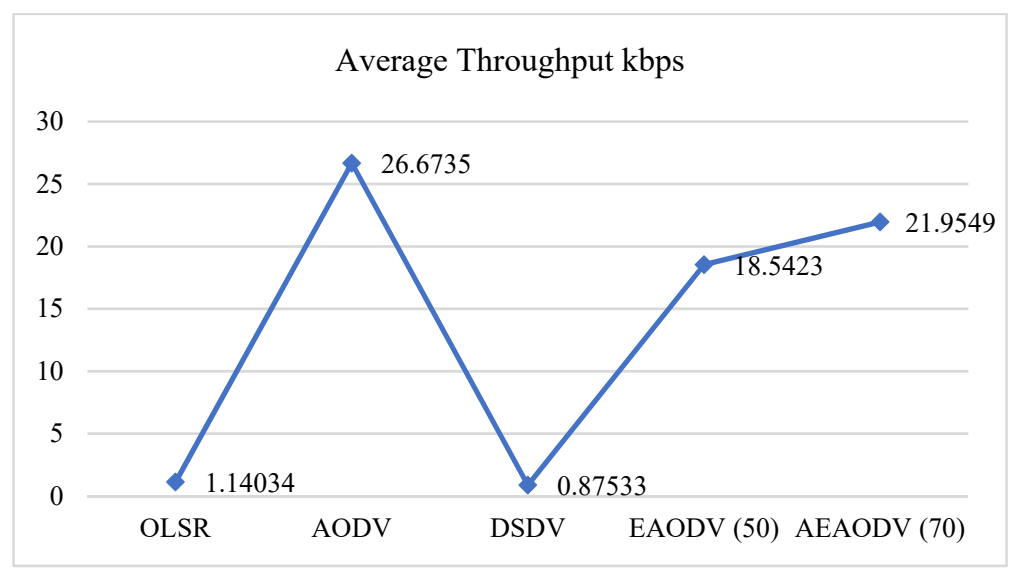

Fig.7. Throughput by AEAODV with other RPs

Fig.7 has shown a comparison of different routing protocols with the proposed AEAODV protocol in terms of throughput (in kbps). The throughput of OLSR is $1.140 \mathrm{kbps}$, AODV is $26.673 \mathrm{kbps}$, DSDV is $0.875 \mathrm{kbps}$, EAODV is $18.542 \mathrm{kbps}$, and AEAODV is $21.954 \mathrm{kbps}$. From this analysis, it is experiential that the existing AODV protocol has improved throughput than the proposed AEAODV and other protocols.

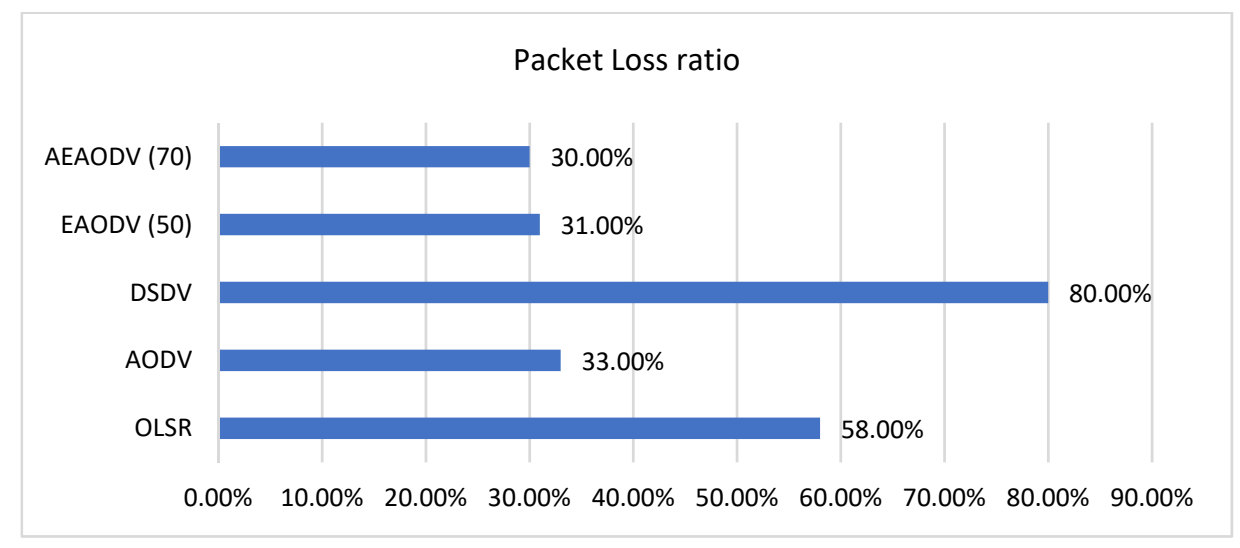


Fig. 8 has shown a comparison of different routing protocols with the proposed AEAODV protocol in terms of PLR (in \%). The PLR of OLSR is $58 \%$, AODV is $33 \%$, DSDV is $80 \%$, EAODV is $31 \%$, and AEAODV is $30 \%$. From this analysis, it is observed that the proposed AEAODV protocol has reduced PLR than other routing protocols.

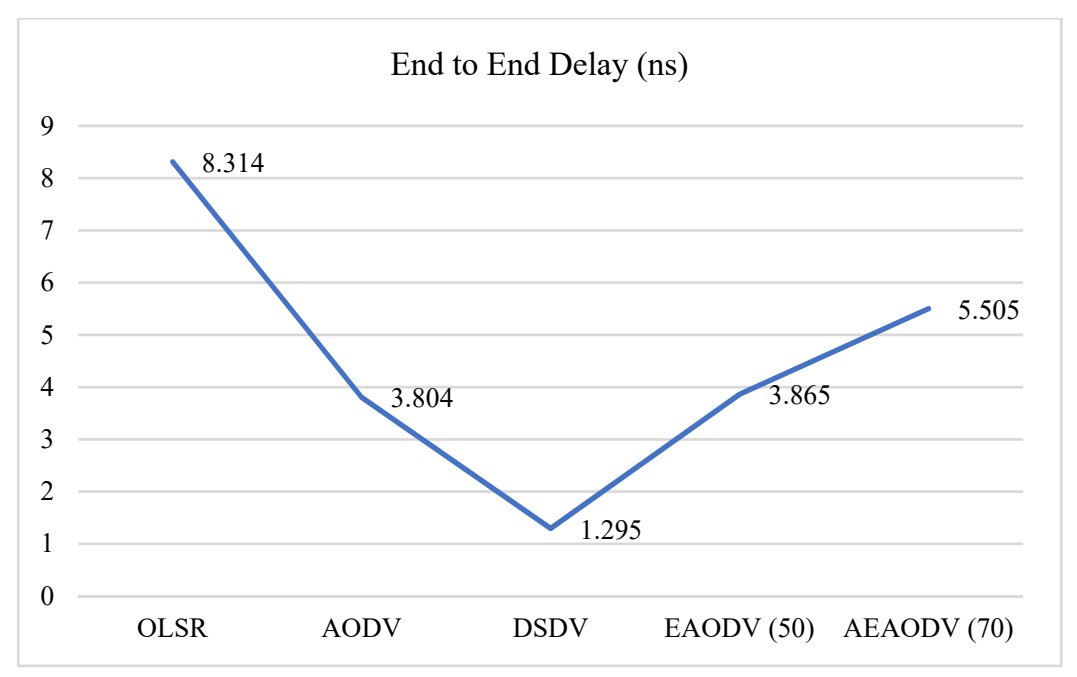

Fig.9. E2E delay by AEAODV with otherRPs

Fig.9 has shown a comparison of different routing protocols with the proposed AEAODV protocol in terms of E2E delay (in nanoseconds). The E2E delay of OLSR is $8.314 \mathrm{~ns}$, AODV is $3.804 \mathrm{~ns}$, DSDV is $1.295 \mathrm{~ns}$, EAODV is $3.865 \mathrm{~ns}$, and AEAODV is $5.505 \mathrm{~ns}$. From this analysis, it is observed that the proposed AEAODV protocol has minimized E2E delay than OLSR protocol but it has a high E2E delay in comparisonto AODV, DSDV, and EAODV routing protocols.

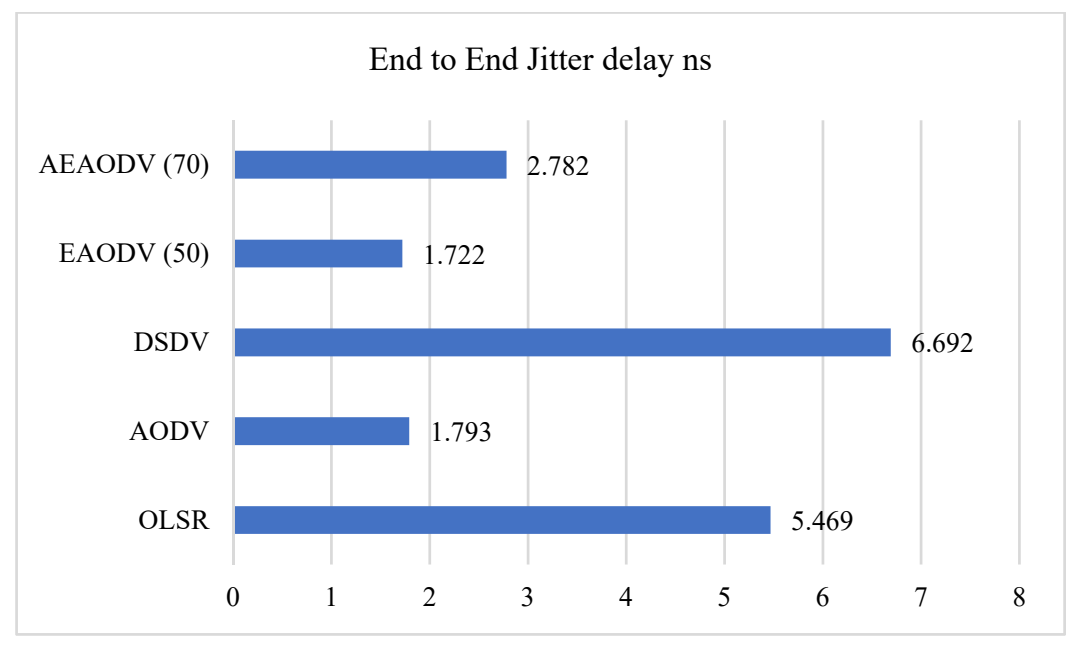

Fig.10. Jitter delay by AEAODV with other RPs

Fig.10 has shown the comparison of different RPs with the proposed AEAODV protocol in terms of E2E Jitter delay (in nanoseconds). The E2E Jitter delay of OLSR is $5.469 \mathrm{~ns}$, AODV is $1.793 \mathrm{ns,} \mathrm{DSDV} \mathrm{is} 6.692 \mathrm{~ns}$, EAODV is $1.722 \mathrm{~ns}$, and AEAODV is $2.782 \mathrm{~ns}$. From this analysis, it is observed that the proposed AEAODV protocol has minimized E2E Jitter delay than OLSR and DSDV protocol but it has a high E2E Jitter delay in comparisonto AODV and EAODV routing protocols. 


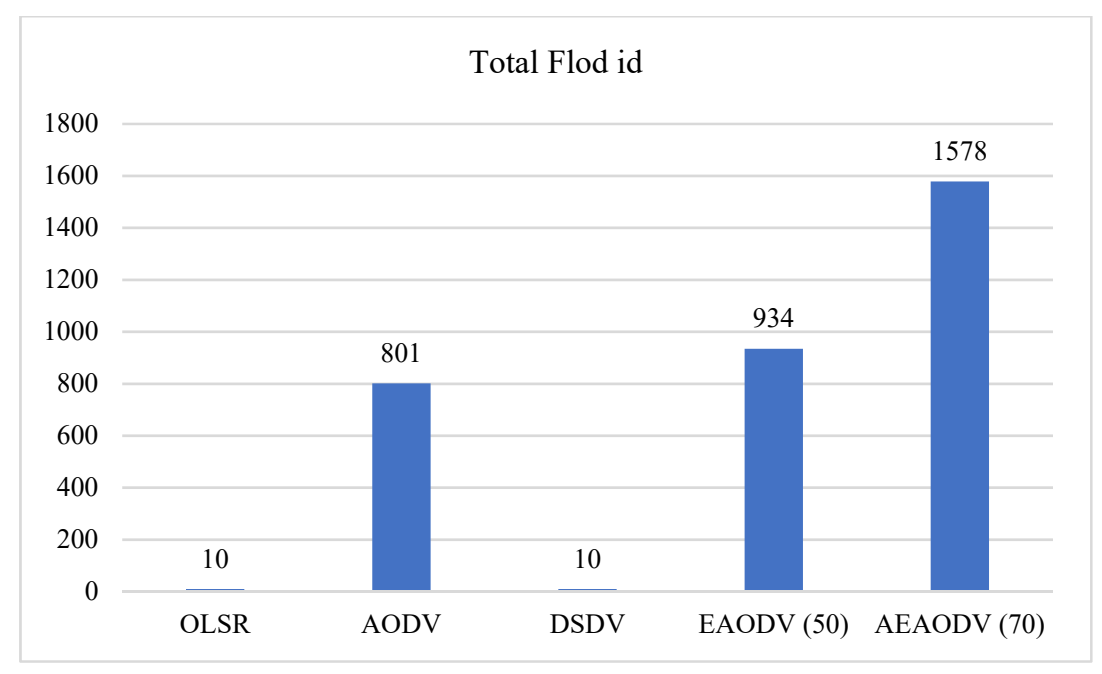

Fig.11. Total Flood_id for AEAODV with otherRPs

Fig. 11 has shown a comparison of different routing protocols with the proposed AEAODV protocol in terms of flood_ids. The number of flood ids for OLSR is 10, AODV is 801, DSDV is 10, EAODV is 934, and AEAODDV is 1578. From this analysis, it is observed that the proposed AEAODV protocol has the highest number of flood_ids than other routing protocols. Here OLSR and DSDV have the same number of flood_ids i.e. 10 .

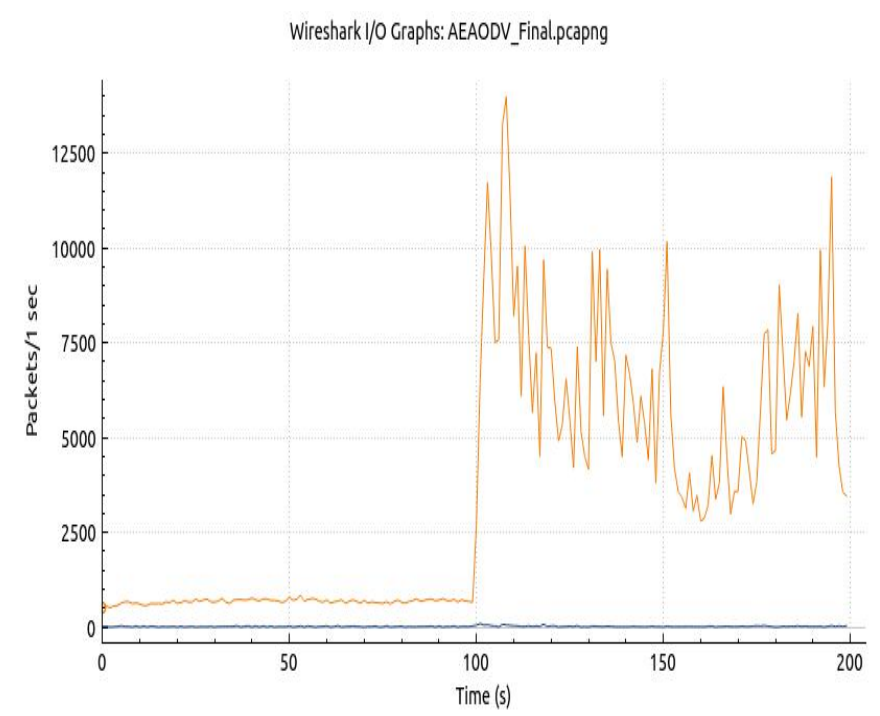

(a) AEAODV

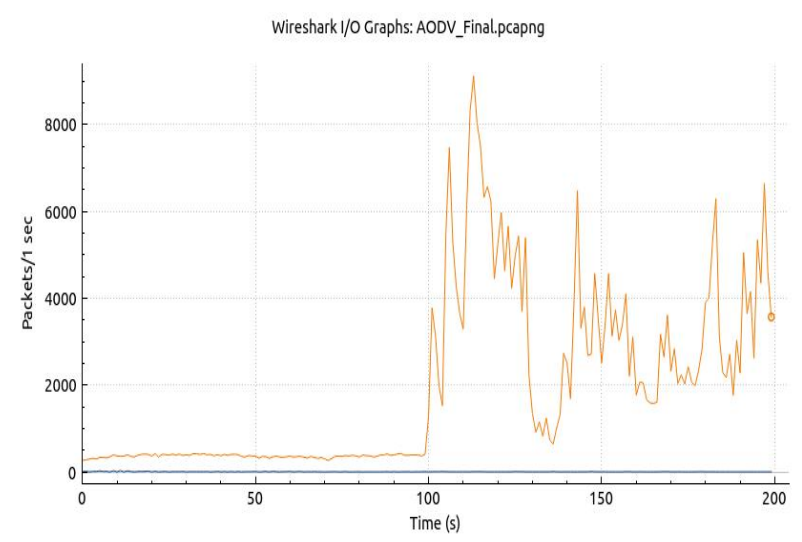

(b) OLSR

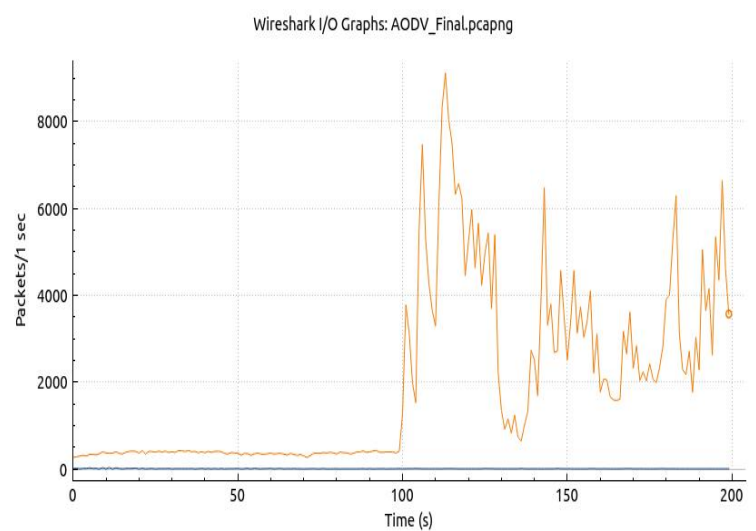

(c) AODV 


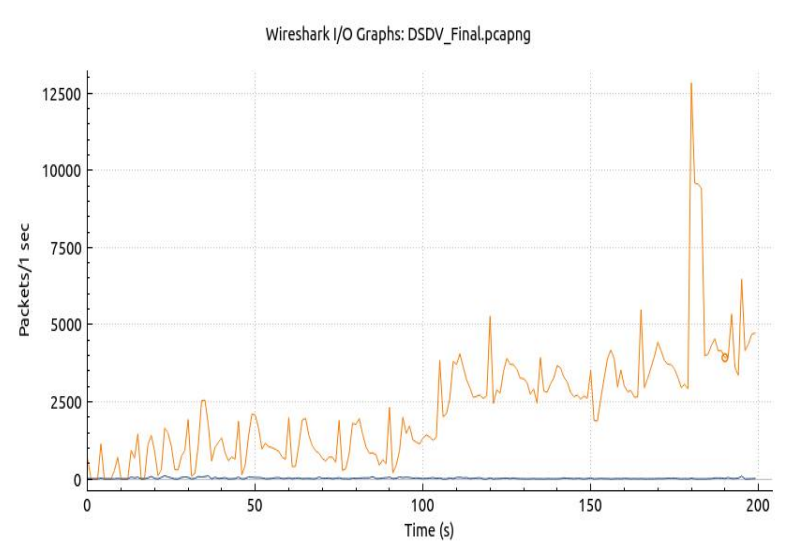

(d) DSDV

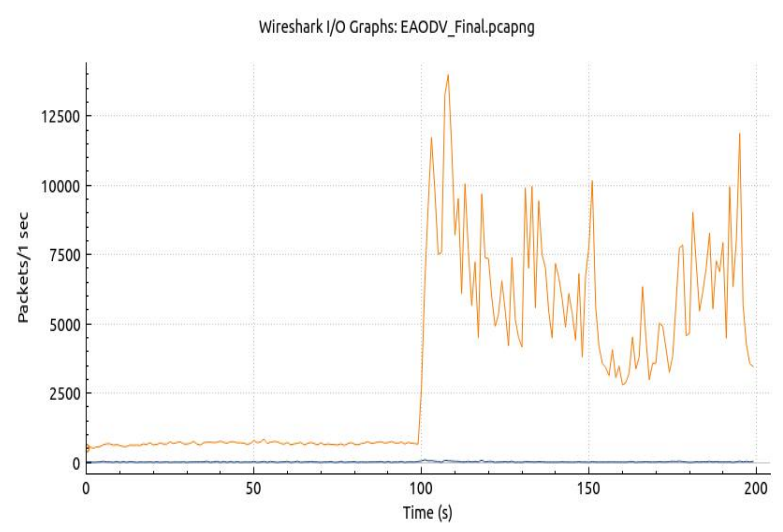

(e) EAODV

Fig.12. Packet transmission rate by AEAODV with other routing protocols

\section{Conclusion}

Due to the greater availability of mobile devices with wireless capability over the last decades, the number of wireless network operators has grown exponentially. MANETS are forms of ad hoc networks, like sensor networks, for non-infrastructure networks. Ad hoc networks also are recognized as temporary networks for implementations in which quick deployment $\&$ information sharing are expected. These networks containmostly a series of mobile nodes, which dynamically modification their positions so that routes from sources to destinations are defined as well as maintained automatically. The presentwork proposed a method that measured fluffyAODV routing protocol.The proposed approach utilizes fluffy rationale procedures to decide a node's trust and incentive by joining the remaining energy, speed of every node in MANET. The outcomes of the simulation show that the improved AEAODV protocol increased network efficiency and effectiveness packet delivery, output as well as decreased overhead or delay in comparison with AODV, DSDV, OLSR as well as EAODVRP.

In our future work, the efficiency of the AEAODV-RP will be improved by defining the required threshold mechanism and examine the energy used with more wireless nodes as well as more kinds of ad hoc network topologies to identify additional metrics that are combined with energy consumption as well as a delivery ratio. We also expect to reduce overhead, interfere with nodes \&improved the network throughput in high density as well as mobility systems.Moreover, we will also try to implement future AEAODV 5G cognitive including IEEE802.16 mesh networks for IoT-based wireless networks.

\section{References}

[1] Ahmad, I. ; ur Rehman, M. (2010): Efficient AODV routing based on traffic load and mobility of node in MANET. 2010 6th International Conference on Emerging Technologies (ICET), Islamabad, Pakistan, pp. 370-375, doi: 10.1109/ICET.2010.5638459.

[2] Akhtar, N.;. Khattak, M. A. K; Ullah, A.; Javed, M. Y.(2017). Efficient routing strategy for congestion avoidance in MANETs. 2017 International Conference on Frontiers of Information Technology (FIT), Islamabad, 2017, pp. 305-309, DOI: 10.1109/FIT.2017.00061

[3] Alex, H.; Michael, N.; Shaoying, Z.; Hussain, A.A. (2013): A Review of routing protocols for mobile ad-hoc networks (MANET). International Journal of Information and Education Technology, 3(1), pp. 1-5, 2013.

[4] Anandha,A. M; M. G.S. (2017) : Cross-layer based congestion detection and routing protocol using fuzzy logic for MANET. Wireless Networks, Springer, 23(5), pp. 1373-1385.

[5] Aouiz, A. A.; Hacene, S. B.; Lorenz, P. (2019): Channel busyness based multipath load balancing routing protocol for ad hoc networks. IEEE Network, 33(5), pp. 118-125, doi: 10.1109/MNET.2019.1900036.

[6] Balakiruthiga, B.; Deepalakshmi, P.; Mohanty, S. N.; Gupta, D.; Kumar, P. P.; Shankar, K.(2020): Segment routing based energy aware routing for software defined data center. Cognitive Systems Research, 64, pp. 146-163, https://doi.org/10.1016/j.cogsys.2020.08.009.

[7] Bamhdi, A. M. (2020) : Efficient dynamic-power AODV routing protocol based on node density. Computer Standards \& Interfaces, 70, , https://doi.org/10.1016/j.csi.2019.103406.

[8] Bento, C.R.D.C.; Wille, E.C. G. (2020):Bio-inspired routing algorithm for MANETs based on fungi networks. Ad Hoc Networks, 107, https://doi.org/10.1016/j.adhoc.2020.102248.

[9] Boukerche, A.; Turgut, B.; Aydin, N. ; Ahmad, M.Z.; Boloni, L. (2011): Turgut. survey paper: routing protocols in ad hoc networks: a survey computer networks. In the International Journal of Computer and Telecommunications Networking, 55(13), pp. 3032-3080.

[10] Chavan, A.A.; Kurule, D.S.; Dere, P.U.(2016). Performance analysis of aodv and dsdv routing protocol in MANET and modifications in aodv against black hole attack. Procedia Computer Science, 79, pp. 835-844, https://doi.org/10.1016/j.procs.2016.03.108.

[11] Ferdous, S. N.; Hossain, M. S. (2016): Randomized energy-based AODV protocol for wireless ad-Hoc network. 2016 3rd International Conference on Electrical Engineering and Information Communication Technology (ICEEICT), Dhaka, pp. 1-5, doi: 10.1109/CEEICT.2016.7873044.

[12] Garg, M. K.; Singh, N.; Rao, M. (2017): Design and implementation of trust-based secure routing protocol for MANETs. IJAER. International Journal of Applied Engineering Research, 12 (16), pp. 5441-5446.

[13] Garg, M. K.; Singh, N.; Verma P. (2018). Fuzzy rule-based approach for design and analysis of a trust-based secure routing protocol for MANETs. Procedia Computer Science, 132, pp. 653-658, https://doi.org/10.1016/j.procs.2018.05.064. 
[14] Kim, B. C.; Lee, H. S. ; Ma,J. S. (2005): Enhanced ad hoc on-demand distance vector (EAODV) routing protocol with route distribution. VTC-2005-Fall. 2005 IEEE 62nd Vehicular Technology Conference, Dallas, TX, USA, pp. 314-318, doi: 10.1109/VETECF.2005.1557523.

[15] Krupa, A.; Benakappa, S. (2010): A survey: routing protocols in MANETs. International Journal of Innovative Research in Computer, 1(3).

[16] Leemaroselin, S (2015): A review on congestion control algorithms in MANET. International Journal of Computer Science \& Engineering Technology (IJCSET), 6( 4), pp. 198-204.

[17] Mamata, R.; Umesh, P. R. ; Niharika, P.; Surendra, K. N.; Sambhu, P. (2017): Congestion control mechanism for real time traffic in mobile adhoc networks. computer communication, Networking and Internet Security, Springer, 5, pp. 149-156.

[18] Murthy, C. ; Ram S. ; Manoj, B. S. (2009): Ad-Hoc wireless networks, architecture and protocols. Pearson Education, Fourth Impression.

[19] Pal, A.; Singh, J. P. ; Dutta, P.(2015): Path length prediction in MANET under AODV routing: Comparative analysis of ARIMA and MLP model, Egyptian Informatics Journal, pp. 103-111, https://doi.org/10.1016/j.eij.2015.01.001.

[20] Perkins, C.; Royer, E.; Das, S. (2003): Ad-hoc On-demand distance vector (aodv) routing. Network Working Group, RFC 3561.

[21] Perkins, C.; Bhagwat, P. (1994): Highly dynamic destination-sequenced distance-vector routing (dsdv) for mobile computers. Proceedings of the Conference on Communications Architectures, Protocols and Applications, pp. 234-244, London, England.

[22] Saba Farheen, N.S.; Jain, A. (2020): Improved routing in MANET with optimized multi path routing fine tuned with hybrid modeling. Journal of King Saud University - Computer and Information Sciences, https://doi.org/10.1016/j.jksuci.2020.01.001

[23] Santosh, K.; Sachin, T. (2015). Energy efficient routing protocol for MANET based on vague set measurement technique. ELSEVIER, 58, pp. 348-355.

[24] Sarkar, D.; Choudhury, S.; Majumder, A. (2018): Enhanced-Ant-AODV for optimal route selection in mobile ad-hoc network. Journal of King Saud University, Computer and Information Sciences, https://doi.org/10.1016/j.jksuci.2018.08.013.

[25] Shukla, A. K.; Jha, C. K. ; Saxena, N.; Biswash, S. K. (2013): The analysis of AODV, based on mobility model. 2013 3rd IEEE International Advance Computing Conference (IACC), Ghaziabad, India, pp. 440-443, doi: 10.1109/IAdCC.2013.6514266.

[26] Singh, Y.; Kumar, A.; Rani, P.; Kaushik, S. K. (2014). Impact of CBR traffic on routing protocols in MANETs, 2014 UKSim-AMSS 16th International Conference on Computer Modelling and Simulation. Cambridge, UK, pp. 474-478, doi: 10.1109/UKSim.2014.91.

[27] Weblink: https://ars.els-cdn.com/content/image/1-s2.0-S1319157812000195-gr3.jpg (Brousing date: May 2021)

[28] Weblink: https://www.researchgate.net/figure/A-Scenario-of-MANET-mobility-management_fig1_221593323 (Brousing date: May 2021).

[29] Yadav, S.; Firdaus, T. (2019): Congestion aware routing in aodv based mobile ad-hoc network (MANET). 2019 International Conference on Computing, Power and Communication Technologies (GUCON), New Delhi, India, pp. 452-457.

\section{AUTHORS PROFILE}

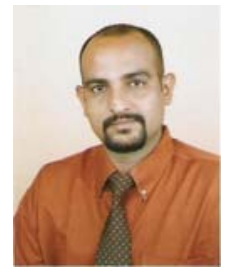

M. Mahto pursued Bachelor of Science from Guru Gasidas University Bilaspur(C.G.), 1989 and Master of Computer Application from Govt. Engineering College Raipur in year 1994. He is currently pursuing Ph.D from Dr. C.V. Raman University Kota Bilaspur (C.G.) and currently working as Programmer in Government Polytechnic Korba under Department of Technical Education Chhattisgarh Raipur, since 1999. He has 22 years of teaching experience and 4 years of other working experience.

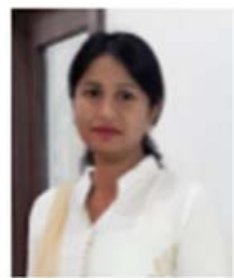

Dr. NeelamSahu obtained Ph.D. degree from Dr. C. V. Raman University Bilaspur(C.G.). She is currently working as a Associate Professor at Dr. C.V. Raman University Kota Bilaspur (C.G.). She has published many papers in national and international journals. 